\title{
Quantum Mechanics, vacuum, particles, Gödel-Cohen incom- pleteness and the Universe
}

\author{
Luis Gonzalez-Mestres ${ }^{1, a}$ \\ ${ }^{1}$ Cosmology Laboratory, John Naisbitt University, Belgrade and Paris \\ Goce Delceva 8, 11070 Novi Beograd, Serbia
}

\begin{abstract}
Are the standard laws of Physics really fundamental principles? Does the physical vacuum have a more primordial internal structure? Are quarks, leptons, gauge bosons... ultimate elementary objects? These three basic questions are actually closely related. If the deep vacuum structure and dynamics turn out to be less trivial than usually depicted, the conventional "elementary" particles will most likely be excitations of such a vacuum dynamics that remains by now unknown. We then expect relativity and quantum mechanics to be low-energy limits of a more fundamental dynamical pattern that generates them at a deeper level. It may even happen that vacuum drives the expansion of the Universe from its own inner dynamics. Inside such a vacuum structure, the speed of light would not be the critical speed for vacuum constituents and propagating signals. The natural scenario would be the superbradyon (superluminal preon) pattern we postulated in 1995, with a new critical speed $c_{s}$ much larger than the speed of light $c$ just as $c$ is much larger than the speed of sound. Superbradyons are assumed to be the bradyons of a super-relativity associated to $c_{s}$ (a Lorentz invariance with $c_{s}$ as the critical speed). Similarly, the standard relativistic space-time with four real coordinates would not necessarily hold beyond low-energy and comparatively local distance scales. Instead, the spinorial space-time (SST) with two complex coordinates we introduced in 1996-97 may be the suitable one to describe the internal structure of vacuum and standard "elementary" particles and, simultaneously, Cosmology at very large distance scales. If the constituents of the preonic vacuum are superluminal, quantum entanglement appears as a natural property provided $c_{s} \gg c$. The value of $c_{s}$ can even be possibly found experimentally by studying entanglement at large distances. It is not excluded that preonic constituents of vacuum can exist in our Universe as free particles ("free" superbradyons), in which case we expect them to be weakly coupled to standard matter. If a preonic vacuum is actually leading the basic dynamics of Particle Physics and Cosmology, and standard particles are vacuum excitations, the Gödel-Cohen incompleteness will apply to vacuum dynamics whereas the conventional laws of physics will actually be approximate and have error bars. We discuss here the possible role of the superbradyonic vacuum and of the SST in generating Quantum Mechanics, as well as the implications of such a dynamical origin of the conventional laws of Physics and possible evidences in experiments and observations. Black holes, gravitational waves, possible "free" superbradyons or preonic waves, unconventional vacuum radiation... are considered from this point of view paying particular attention to LIGO, VIRGO and CERN experiments.
\end{abstract}

\section{This lecture is dedicated to the memory of John Bell}

\footnotetext{
ae-mail: luis.gonzalez-mestres@cosmology.megatrend.edu.rs;personale-mail:lgmsci@yahoo.fr
} 


\section{Introduction}

Is Quantum Mechanics an ultimate principle of Physics, or an approximate property of standard particles generated at a more fundamental level by a deeper dynamics?

Quantum Mechanics (QM) is usually assumed to be a basic principle of standard physics, and plays a central role in the description of conventional "elementary" particles. In particular, Quantum Field Theory (QFT) successfully describes many aspects of particle physics, and has generated the "standard model" of electro-weak interactions as well as Quantum Chromodynamics (QCD). No deviation from Quantum Mechanics has by now been demonstrated experimentally.

In spite of such evidences, the question of the foundations of Quantum Mechanics is far from being just an academic one. It has a direct interest for practical applications in electronics, computers and other industrial fields as well as in various domains of fundamental research.

\subsection{The uncertainties about entanglement}

Quantum entanglement plays a crucial role in the theory of quantum information, including quantum teleportation that is claimed to be successful up to distances of $\simeq 100 \mathrm{Km} \mathrm{[1]} \mathrm{and} \mathrm{quantum} \mathrm{key}$ distribution (QKD) where a system able to operate over $307 \mathrm{Km}$ of optical fibre has been described [2]. More recently, the results of measurement device independent QKD (MDIQKD) over $404 \mathrm{~km}$ of ultralow-loss optical fibre and $311 \mathrm{~km}$ of standard optical fibre have been reported [3].

Simultaneously, quantum telescopes $[4,5]$ and other uses of entanglement are being seriously considered. A satellite devoted to test the transfer of quantum information between space and Earth has also been launched $[6,7]$.

In all cases, it is hoped that Quantum Mechanics remains valid at the relevant distance scales. On this fundamental question, further work is required.

Understanding the deep origin of Quantum Mechanics can help to better determine its validity and possible modifications, and to develop all the above mentioned fields.

Standard quantum mechanics has often been considered as counter-intuitive, in spite of experimental evidence [8]. Entanglement appears as a mysterious phenomenon from the point of view of relativity, as no signal is expected to travel faster than light.

Direct experimental tests of quantum entanglement, involving a small time interval comptatible with space-like separation between two distant local events, have been performed up to distances of $1.3 \mathrm{Km}$, confirming the validity of QM theory at this scale. $[9,10]$. This is the largest distance scale at which entanglement has been direcly checked in this way until now.

\subsection{On alternatives to standard theories}

Although there is no practical incompatibility between relativity and Quantum Mechanics for the description of standard matter and QFT is a successful approach, the combination of both theories has nontrivial implications such as excluding most local hidden variable approaches to QM $[9,10]$.

A major challenge for QFT is the elaboration of a quantum theory of gravity [11, 12]. In the standard approach, quantum gravity is usually expected to provide an ultimate theory for both particle physics and Big Bang cosmology. Such a point of view can, however, be questioned as new physics 
(preons...) may manifest itself at very high energy and very small time and distance scales [13, 14]. Quantum Mechanics and relativity are then in most cases expected to be of composite origin [15, 16].

As emphasized in $[14,15]$, and already considered in $[17,18]$, the most relevant preonic pattern to generate possible violations of standard relativity and Quantum Mechanics would be a nontrivial internal structure of the physical vacuum. Contrary to the original preon model [20] where preons were assumed to be direct constituents of the standard particles, the basic preon model dealt with in $[17,18]$ and in $[14,15]$ assumes vacuum to be made of preonic constituents and standard particles to be excitations of such a vacuum (see also [19]). Conventional relativity and Quantum Mechanics become then low-energy limits of a more sophisticated and fundamental dynamics.

The existence of a pre-Big Bang era would also be a natural consequence of this, yet unexplored, preonic vacuum structure [21, 22]. In such a scenario, the standard Big Bang must be replaced by a new cosmology at the early stages and cosmic inflation is no longer necessary [13, 23].

\subsection{The possible role of space-time structure}

The structure of space-time is also a crucial issue to understand the possible origin of Quantum Mechanics $[14,15]$. In particular, nothing is known about the properties of space-time inside the physical vacuum. Just as the speed of sound is much smaller than the critical speed of the particles forming a solid, it seems natural to assume $[17,18]$ that the critical speed $c_{s}$ of the preonic constituents of vacuum is much larger than that the speed of light $c$. Then, the propagation of superluminal signals in vacuum would provide a natural explanation for the observed quantum entanglement $[14,15]$ and, simultaneously, a possible large distance limit for the validity of Quantum Mechanics.

Furthermore, the space-time structure at this deeper level can also go beyond the standard real space-time coordinates and their conventional extensions. In [24, 25], we introduced a spinorial spacetime (SST) where two complex coordinates replace the four real ones with the symmetry group SU(2) instead of the usual $\mathrm{SO}(3)$ for space rotations. Spin-1/2 particles become then actual representations of the space symmetry group, and it is tempting to consider a possible relation between the SU(2) complex space-time coordinates and the complex wave functions of Quantum Mechanics [14, 15].

The SST cosmic geometry locally predicts the existence of a privileged space direction (PSD) for each comoving observer as already noticed in [24, 25] and further discussed in [26, 27] as well as in more recent work [13, 23]. It must be emphasized, however, that this property does not by itself imply any anisotropy in the global expansion of the Universe. The existence of the PSD may have been confirmed by Planck data $[28,29]$. But such a PSD is strictly a local phenomenon and the SST defines a globally uniform and isotropic cosmic geometry. Thus, a cosmic space-time geometry based on the SST appears to be consistent with Planck results and simultaneously with recent work confirming the isotropy of the expansion of the Universe [30].

\subsection{A new approach to Quantum Mechanics}

As discussed in $[14,15],[13,23]$ and in previous papers, the SST geometry and a preonic vacuum structure can be reliably combined to build an alternative approach to Quantum Mechanics. And, subsequently, to the Big Bang cosmology based on conventional QM and relativity at scales where the validity of these principles has not really been tested .

In this lecture, we present un updated detailed discussion of such a pattern, including possible experimental and cosmological tests as well as the implications of Gödel-Cohen incompleteness [31]. 


\section{Bell's theorems and experimental tests}

What would be the implications of strict standard relativistic causality for the theoretical and phenomenological content, origin and implications of Quantum Mechanics?

The original theorems $(1964,1975)$ by John Stuart Bell $[32,33]$ have plaid a crucial role in the understanding of the grounds of QM and in the conception of possible experimental tests of its basic propeties. They still lead nowadays to interesting conceptual controversies [34, 35].

John Bell actually taught us what is, and what is not, Quantum Mechanics and how one can test the validity of standard QM. Bell's work and life are remembered in [36, 37].

\subsection{Bell's work and its relevance}

How to conceive experiments allowing to confirm the validity of standard quantum mechanics and to exclude interpretations based on local hidden variables? Bell's inequalities have clearly answered the theoretical side of this question and opened the way to successful experimental tests.

The first paper by John Bell (1964), On The Einstein Podolsky Rosen Paradox, refers to the 1935 article by Albert Einstein, Boris Podolsky and Nathan Rosen (EPR) Can Quantum-Mechanical Description of Physical Reality Be Considered Complete? [38]. The end of the EPR abstract explicitly states on the properties of quantum wave functions:

"One is thus led to conclude that the description of reality as given by a wave function is not complete".

In [32], Bell subsequently cites this point of wiew by Albert Einstein [39]:

"But on one supposition we should, in my opinion, hold fast: the real factual situation of the system $S_{2}$ is independent of what is done with the system $S_{1}$, which is spatially separated from the former".

To refute the arguments of $[38,39]$ Bell wrote in particular in 1964:

"In a theory in which parameters are added to quantum mechanics to determine the results of individual measurements, without changing the statistical predictions, there must be a mechanism whereby the setting of one measuring device can influence the reading of another instrument, however remote. Moreover, the signal involved must propagate instantaneously, so that such a theory could not be Lorentz invariant".

Later, in his book Speakable and Unspeakable in Quantum Mechanics [40], commenting on a hidden variable approach to Quantum Mechanics considered by Georges Lochak [41], John Bell wrote explicitly:

"... if his extension is local it will not agree with quantum mechanics, and if it agrees with quantum mechanics it will not be local".

This was the main result of John Bell's work that recent loophole-free experiments appear to have clearly confirmed more than 50 years after his first pioneering article. To reach such an experimental accomplishment, a remarkable long-term effort has been necessary.

\subsection{Bell's inequalities}

In what follows, to discuss the basic content of Bell's inequalities and the associated experiments, we refer to the CHSH formulation [42]. The CHSH approach to Bell's theorems is used in $[9,44]$ and also in [45] and in previous tests of Bell's inequalities. We also assume that the recent experiments presented as "loophole-free" have really this property. 
In the CHSH formulation of Bell's result, the test of the possible existence of local hidden variables with standard relativity uses two boxes, $\mathrm{A}$ and $\mathrm{B}$, able to receive a binary input ( 0 or 1$)$ and produce a binary output $(+1$ or -1$)$. We are interested in the expectation value $\langle x . y\rangle_{(a, b)}$ where $x . y$ stands for the product of the output values ( $x$ for box A, $y$ for box $B$ ) given the random inputs $a$ for box A and $b$ for box B. Then, the "local realism" discussed by John Bell leads to:

$$
S=\left\langle x \cdot y>_{(0,0)}+<x \cdot y>_{(0,1)}+<x \cdot y>_{(1,0)}-<x \cdot y>_{(1,1)} \leq 2\right.
$$

The value $S=0$ is obtained if $x$ and $y$ take random values. If $x$ and $y$ are both systematically equal to 1 (or to -1), one gets $S=2$. Values of $S$ larger than 2 are obtained introducing correlations between $x$ and $y$ contrary to the locality assumption. Measuring experimentally the value of $S$ and of similar quantities emerging from Bell's work is therefore a very important task.

Since the 1970s, experimentalists worked to test Bell inequalities [46] and the results confirmed their violation as expected from standard Quantum Mechanics. However, the existence of various possible loopholes was often claimed [47]. To face these criticisms, experiments explicitly presented as loophole-free have been performed in the recent years [43].

\subsection{Loophole-free experiments}

In particular, the result of a loophole-free Bell test involving a comparatively large distance scale (1.3 $\mathrm{Km})$ has recently been reported $[9,10]$.

Using an experimental scheme with high-fidelity entanglement between distant electron spins, the measurements described in [9] get $S=2.42 \pm 0.20$. The time window during which $\mathrm{A}$ and $\mathrm{B}$ are space-like separated and measurements can be performed is $4.27 \mu \mathrm{s}$. A second experiment with minor changes [10] has more recently yielded $S=2.35 \pm 0.18$.

Simultaneously, loophole-free experiments using entangled photon pairs [44, 45] yield equally results against the hypothesis of local realism.

The results of $[9,10]$ and of $[44,45]$ appear to be clearly incompatible with most conventional local hidden variable interpretations of Quantum Mechanics based on standard relativity. However, the authors of [9] clearly emphasize that: i) their work "rules out large classes of local realist theories"; ii) "no Bell experiment can exclude the infinite number of local realist theories". To these comments, [10] adds a conclusion claiming that the second data run has "strengthened the rejection of a broad class of local realistic theories". A more recent test of Bell inequalities can be found in [48].

As remarkably foreseen and explicitly developed by John Bell, the measurement of $S$ and of related parameters has been shown to be a direct, efficient way to test standard QM and exclude most local hidden variable approaches provided loopholes can be ruled out.

\section{Beyond Bell's theorems}

What can be the future of Quantum Mechanics after the successful loophole-free tests of the expected violation of Bell's inequalities?

As underlined in $[9,10]$, local hidden variables cannot be totally excluded by the recent and experimental tests of Bell's inequalities. Furthermore, Bell's theorems assumed special relativity to hold exactly and to be a universal law. There is obviously room for further studies, including possible new physics, without contradicting the successful use of the work of John Bell to check experimentally the specific properties of standard QM. 
In particular, the confirmed violation of Bell's inequalities does not contradict the possibility that Quantum Mechanics be of composite origin, emerging from a more fundamental set of laws. Conventional QM is not incomplete as a physical law, but it can actually be an approximation holding with some limitations for standard particles at low energy $[14,15]$.

Bell's inequalities were obtained assuming locality and standard relativity, without considering any possible internal structure of the physical vacuum. Going beyond such a scenario appears today as a natural option $[13,23]$.

In particular, the time window of $4.27 \mu s$ during which A and B are space-like separated in [9] corresponds to the distance of $1.28 \mathrm{Km}$ divided by the speed of light $c$. This allows to exclude a standard relativistic exchange between the two boxes. But if the physical vacuum is made of superluminal preons, as considered in $[17,18]$ and in subsequent papers, the propagation of superluminal signals in vacuum can generate a new kind of causal connection between A and B without contradicting the results of $[9,10]$ and of the other loophole-free experiments that have confirmed the violation of Bell's inequalities for conventional particles with standard relativity.

A natural question is then: why would the superluminal signal propagation inside the physical vacuum act on the internal structure and properties of standard particles in such a way that quantum entanglement can be generated? A logical answer is that in such an approach the conventional "elementary" particles are actually excitations of the physical vacuum. Then, in a sense, two entangled particles form a single vacuum excitation at the time scales of current experiments.

\subsection{Quantization and complex wave functions}

Interpreting standard particles as excitations of vacuum raises the basic questions of origin of quantization and of the generation of complex wave functions. Natural quantization and Fermi statistics for spinorial wave functions, leading subsequently to quantization and Bose statistics for composite bosons, was considered in $[14,15]$ and in $[13,23]$. If standard particles are just solutions of the vacuum internal equations, Fermi statistics naturally emerges if such equations lead to spinorial wave functions as the solutions at the basic level, and if these solutions are unique around each point of space-time for each relevant set of quantum numbers. The standard exclusion principle would then just reflect the uniqueness of the solutions to vacuum equations.

As discussed in $[14,15]$ and in $[13,23]$, complex wave functions and Fermi quantization can automatically emerge from the fundamental equations of a vacuum with a SST. Space-time coordinates would then be complex and spinorial wave functions would naturally correspond to the structure of the spinorial space-time. Fermi statistics would just reflect the existence of a single local solution to the basic dynamical equations for each kind of spinorial excitations, leading to the observed quantization. Bosons can be obtained as composite objects made of two fermions, or as new solutions to vacuum equations subject to similar constraints. A scalar complex wave function can be the product of two complex spinors from which the scalar object is made.

In such an approach, as in standard Quantum Mechanics, quantum uncertainty would be a natural consequence of the wave nature of the particles generated as vacuum excitations. Precision experiments [49] do not seem to confirm the "measurement-disturbance relationship" suggested by Werner Heisenberg [50]. In 1927, Heisenberg wrote in particular [51]:

"At the instant of time when the position is determined, that is, at the instant when the photon is scattered by the electron, the electron undergoes a discontinuous change in momentum. This change is the greater the smaller the wavelength of the light employed, i.e., the more exact the determination of the position". 
But reference [49] concludes, on the grounds of experimental results, that "although correct for uncertainties in states, the form of Heisenberg's precision limit is incorrect if naively applied to measurement".

More generally, in connection with the possible origin of wave functions, the existence of complex soliton solutions with real energy to complexified classical equations (Korteweg - de Vries, SineGordon...) must also be noticed [52].

\section{A possible origin of Quantum Mechanics}

In $[14,15],[53,54],[13,55]$ and in other papers, we explicitly considered the possible generation of Quantum Mechanics from a preonic vacuum with a SST space-time geometry. In [14], we illustrated this idea using cosmic spinorial coordinates $\left(\xi, \xi_{0}\right)$ and writing a classical wave function for a spin-1/2 particle at rest around a space-time origin $\xi_{0}$ of the form:

$$
\Psi_{s p}(\xi)=F\left(\left|\xi-\xi_{0}\right|^{2}\right)\left(\xi-\xi_{0}\right)
$$

together with:

$$
\Psi_{s p}^{*}(\xi)=F\left(\left|\xi-\xi_{0}\right|^{2}\right)\left(\xi-\xi_{0}\right)^{*}
$$

where * stands for complex conjugate corresponding to opposite spin. The real function $F$ is assumed to contain an appropriate cutoff in $\left|\xi-\xi_{0}\right|^{2}$.

It was emphasized in [14] that the wave functions $\Psi_{s p}$ and $\Psi_{s p}^{*}$ violate local standard causality, as they take nonzero values for present and future values of cosmic time around $\left|\xi_{0}\right|$ (see next Section). Space-like distances are thus replaced by direct spinorial distances on the SST. $F$ tacitly defines a space-time distance scale $\lambda_{S S T}$ below which causality does no longer hold in the conventional sense used for the standard relativistic space-time.

As indicated before and suggested in previous papers, Quantum Mechanics and Fermi statistics can naturally be generated if $\Psi_{s p}$ and $\Psi_{s p}^{*}$ provide indeed a unique solution to the internal equations of a preonic physical vacuum for the relevant set of quantum numbers.

The expression $\left|\xi-\xi_{0}\right|$ actually corresponds to a local time scale that will be associated by dynamics to a relevant space scale. In any case, as the upper bounds on the size of the electron are still far from the Planck length, the dynamical structure of expressions like (2) and (3) can manifest itself well before Planck scale introducing new physics for standard "elementary" particles. The study of ultra-high energy cosmic rays (UHECR) can thus play a crucial role to search for deformations and violations of standard relativity and quantum mechanics generated by a new dynamics [56, 57]. Experiments like AUGER [58] can yield important results in this respect [59, 60].

\subsection{SST and the propagation of extended objects}

An additional mechanism can contribute to the inset of Quantum Mechanics, as discussed in [15, 53], $[54,55]$, and later in this lecture. For a given value of $\xi_{0}$, the spinorial particle structure defined by equations like (2) and (3) is not only extended in space but also in time.

As a result, the time propagation of a spin-1/2 particle with an internal structure defined by (2) or (3) generates an overlap between wave functions corresponding to different values of $\xi_{0}$ and of the cosmic time $\left|\xi_{0}\right|$. Thus, a continuously propagating particle is not, strictly speaking, a solution of the vacuum equations that generate the static solutions (2) and (3). Continuous propagation of such an extended object can be forbidden for this reason. 
A natural solution to avoid this difficulty would be a discrete, and basically random, propagation of particles at very small time scales. The situation will then look close to that generating the Feynman path integral of standard quantum mechanics at larger scales. Thus, QM will emerge as an approximate large distance and low energy description of a more involved dynamics.

\subsection{Particle wave functions and the physical vacuum}

Clearly, the hypothesis that $\Psi_{s p}$ and $\Psi_{s p}^{*}$ describe excitations of the inner vacuum dynamics is a natural one and can reasonably challenge the standard QFT approach where all the dynamics is carried by the "elementary" particles and the vacuum is basically empty except for the condensates of conventional particle fields related to spontaneous symmetry breaking and for the zero modes of boson fields.

Conventional QFT particles do not interact with any inner vacuum dynamics. But the established validity domain of such a quantum field theory is not larger than that covered by accelerator experiments. Thus, new physics involving a direct role of the physical vacuum may manifest itself at higher energy scales. The generation of Quantum Mechanics from vacuum dynamics would basically occur at very small distance scales, largely beyond the reach of LHC experiments even if some signatures at accelerators can be imagined as discussed in Section 10.

If fermions are generated through equations like (2) and (3) corresponding to unique solutions satisfying the requirements of vacuum internal dynamics (see also Subsection 3.1), and the harmonicoscillator nature of standard boson fields allows for alternative descriptions concerning the presence of bosonic zero modes in vacuum [26,61], possible solutions to the standard cosmological constant problem naturally emerge. Understanding the deep nature of vacuum as well as its actual relation with the dynamics of our Universe and of standard matter is then a crucial subject on which complementary material has been presented in [62].

\subsection{Superluminal signals, quantum dynamics and Cosmology}

Quantum entanglement is not the only phenomenon of quantum physics where superluminal signals can be present. In [61, 63] and in [26], we considered the possibility that the standard condensates and bosonic zero modes of QFT would actually not be permanently present in a superbradyonic vacuum. This can be possible if, as a conventional particle (vacuum excitation) gets close to the local region considered, the vacuum constituents react in order to temporarily generate the relevant bosonic components allowing the particle to propagate and interact.

In such a mechanism, a superluminal signal emitted by the approaching particle can play an important role reaching the vacuum structure in the relevant region and activating its dynamical reorganization in order to allow the particle to propagate and interact. This nonstandard dynamics would be difficult to observe in laboratory experiments because of the permanent presence of surrounding matter, but can play a crucial role at cosmic level.

In all cases, the generation of particles and Quantum Mechanics from the vacuum inner structure would open the way to new dynamical potentialities that are never considered in standard particle phyisics and QFT. If particles are actually vacuum excitations and Quantum Mechanics is generated by vacuum dynamics, a new cosmology should be built $[13,26]$ : instead of a Universe "made of particles", the relevant subject would be the expanding vacuum together with its excitations $[64,65]$.

If Quantum Mechanics is not a fundamental principle of Physics but results from the dynamics of the physical vacuum, a pre-Big Bang phase of the history of the Universe is obviously required $[13,26]$ beyond the "primeval quanta" postulated in 1931 by Georges Lemaître as The Beginning of the World [66]. Since then, Physics has evolved and new concepts have been developed. 


\section{The spinorial space-time}

We briefly remind and update here part of the material presented in previous contributions to this conference (see also [53, 67]). The SST appears as the natural space-time geometry to describe a physical world where fermions exist with spinorial wave functions that should be actual representations of the space symmetry group. The implications of the SST geometry for Particle Physics and Cosmology turn out to be surprisingly strong and deserve careful attention.

In particular, the cosmic SST automatically yields the $H t=1$ relation $(H=$ ratio between relative velocities and distances at cosmic level, $t=$ age of the Universe) in a purely geometric way, without requiring any explicit presence of conventional matter and of the associated dynamics. This property can substantially change the formulation of cosmological laws

The spinorial space-time introduced in $[24,25]$ is a basic $S U(2)$ representation with two complex coordinates replacing the standard four real ones of the relativistic space-time. Its properties, with possible cosmological implications directly generated by its mathematical structure, have been further considered in subsequent papers including [26, 27], [68, 69] and [13, 23].

\subsection{SST, cosmic time, age of the Universe}

To a SU(2) spinor $\xi$ describing the cosmic SST coordinates (two complex variables instead of the conventional four real ones) of a point of our space-time, it is possible to associate a positive SU(2) scalar $|\xi|$ defined by the relation $|\xi|^{2}=\xi^{\dagger} \xi$ (the dagger stands for hermitic conjugate).

Then, a suitable definition of the cosmic time naturally equivalent to the age of the Universe can be $t=|\xi|$. The associated space is given by the $S^{3}$ hypersphere $|\xi|=t$ with an additional spinorial structure that does not exist in the standard space. Other definitions of the cosmic time $t$ in terms of $|\xi|$ (f.i. $t=|\xi|^{2}$ ) can also be used, but they lead to similar cosmological results as long as a single-valued function of $|\xi|$ provides the definition of the cosmic time.

In this definition of the age of the Universe, the value $t=0$ is reduced to a point. There can actually be some nontrivial structure around this value in the real history of the Universe, but the relevant values of its size in space and time would be very small and can be neglected in practice except for specific studies of the initial singularity.

\subsection{SST, cosmic coordinates and expanding Universe}

Using the definition $t=|\xi|$, and taking $\xi_{0}$ to be the observer position on the $|\xi|=t_{0}$ hypersphere, space translations can be defined inside this associated space hypersphere (the space at $t=\left|\xi_{0}\right|$ ) and simultaneously on all the space hyperspheres of the SST by SU(2) transformations acting on the whole cosmic spinor space. One can then write $\xi=U \xi_{0}$ with:

$$
U=\exp \left(i / 2 t_{0}^{-1} \vec{\sigma} \cdot \overrightarrow{\mathbf{x}}\right) \equiv U(\overrightarrow{\mathbf{x}})
$$

where $\vec{\sigma}$ is the vector formed by the usual Pauli matrices and the vector $\overrightarrow{\mathbf{x}}$ the spatial position (in time units, at the present stage) of $\xi$ with respect to $\xi_{0}$ at constant cosmic time $t_{0}$ as measured on the $S^{3}$ hypersphere. Equation (4) tacitly implies that $\overrightarrow{\mathbf{x}}$ must be proportional to $t_{0}$.

The origin of cosmic time, naturally associated to the beginning of the Universe, is given by the point $\xi=0$ where, as just stressed, the initial space is contracted to a single point. One then gets an expanding universe where cosmological comoving frames correspond to straight lines in the SST starting from the time origin $\xi=0$ and are transformed into eachother under the action of the cosmic 
SU(2) group. Thus, the cosmic SST geometry naturally provides for each comoving observer a local privileged rest frame corresponding to the relevant straight line $\left(\xi_{0}=t\right.$ times a constant spinor) in a way compatible with existing cosmological observations.

As already noticed in $[24,25]$, attempts to associate to the cosmic spinor $\xi$ a space-like position vector with real cosmic space coordinates defined by $\overrightarrow{\mathbf{x}_{c}}=\xi^{\dagger} \vec{\sigma} \xi$ do not actually generate such spatial coordinates. Instead, one gets $|\xi|^{2}$ times a unit vector corresponding to a local privileged space direction (PSD) "parallel" (in the SST) to the cosmic spinor $\xi$. The direction in the $S^{3}$ hypersphere associated to the vector $\xi^{\dagger} \vec{\sigma} \xi$ corresponds to the set of points whose cosmic SST space-time position is equal to $\xi$ times a complex phase.

Thus, to define the standard real space coordinates in the SST, a space origin $\xi_{0}$ at the cosmic time $t_{0}=\left|\xi_{0}\right|$ is required, as in equation (1). Such coordinates correspond to a local description of the $S^{3}$ hypersphere at cosmic time $t_{0}$ as viewed from the space origin $\xi_{0}$.

The new space-time geometry introduced by the SST clearly leads to natural limitations for general relativity and standard cosmology. Rather than an intrinsic fundamental property of space and time defining a global space-time geometry, conventional relativity is expected to be a low-energy symmetry of standard matter similar to the effective Lorentz-like symmetry of the kinematics of lowmomentum phonons or solitons in a condensed medium [17, 18]. In condensed matter, the speed of sound or the maximum speed of solitons plays the role of the critical speed. The speed of light $c$ would then be the critical speed of a family of excitations of the physical vacuum (the standard particles) not directly associated to an intrinsic geometry of space-time in our Universe.

Space rotations around a fixed point $\xi_{0}$ in the SST at $t=t_{0}$ are given by SU(2) transformations acting on the spatial position vector $\overrightarrow{\mathbf{x}}$ defined by (1). A standard spatial rotation with respect to the local origin $\xi_{0}$ is now given by a SU(2) element $U(\overrightarrow{\mathbf{y}})$ turning $U(\overrightarrow{\mathbf{x}})$ into $U(\overrightarrow{\mathbf{y}}) U(\overrightarrow{\mathbf{x}}) U(\overrightarrow{\mathbf{y}})^{\dagger}$. The vector $\overrightarrow{\mathbf{y}}$, related to $U(\overrightarrow{\mathbf{y}})$ in a similar way to (1), provides the rotation axis and angle. If a spin-1/2 object is present at the position $\overrightarrow{\mathbf{x}}$ with an associated local spinor coordinate $\xi_{p}$ describing its internal structure, then $\xi_{p}$ transforms into $\xi_{p}^{\prime}=U(\overrightarrow{\mathbf{y}}) \xi_{p}$.

\subsection{Some properties of the SST Universe}

The cosmic SST just described automatically generates three basic cosmological phenomena in a purely geometric way $[26,27]$ and without any explicit presence of matter and energy:

i) The standard Lundmark-Lemaître-Hubble (LLH) relation between relative velocities and distances at cosmic scale, with a ratio $H$ (velocity/distance) equal to the inverse of the age of the Universe $\left(H=t^{-1}\right)$. As $t$ is the radius of the spatial hypersphere, and comoving frames correspond to straight lines starting from the time origin, distances between comoving objects will be proportional to $t$ and the (constant) associated speeds will be given by these distances divided by $t$. Thus, $t^{-1}$ turns out to be the automatic geometric value of the LLH constant $H$ in the SST.

ii) The existence of a privileged space direction (PSD) for each comoving observer, as already obtained in [24, 25] and further studied in [26, 27] and in subsequent papers. As previously stressed, the existence of such a local PSD does not imply any global anisotropy in the expansion of the Universe. The global expansion is totally isotropic on purely geometric grounds, and space remains a homogeneous hypersphere for all values of $t$.

iii) In the direct SST formulation, space translations form a (non-abelian) compact group, contrary to standard space-time. This allows to escape standard no-go theorems for symmetries.

More details, including a study of the cosmological implications of the unconventional properties of the cosmic SST, are given in [26, 27], [68, 69], [13, 23] and in related papers. 
In all cases, a fundamental feature of the cosmic spinorial space-time geometry is that it is not dominated by standard matter or conventional dark energy, and that its structure is defined in a totally independent way suggesting a more primordial origin (vacuum dynamics?). In the SST without standard matter, space units are not required, as time provides an effective distance scale.

The possible connection between the cosmic SST structure and the ultimate dynamics of matter and vacuum is a crucial open question requiring further fundamental research (theory, experiment, observation, phenomenology). Preonic and and pre-Big Bang scenarios should be carefully considered in this respect $[13,15]$. Furthermore, the definition of the direction of time with the SST geometry leads to a natural contradiction between local and cosmic levels.

\subsection{SST and the space-time contradiction}

What would be the dynamical implications of introducing a local SST geometry around a space-time origin $\xi_{0}$ different from the cosmic origin $\xi=0$ ?

As discussed in [15] and previously in [53, 54], introducing a local SST (the above considered wave functions are just an example) automatically leads to a contradiction between the time and space directions defined at $\xi=\xi_{0}$ by the cosmic SST $(t=|\xi|)$ and the structure generated by the local SST where time for $\xi$ would be defined by $t_{\text {local }}=\left|\xi-\xi_{0}\right|$.

Similarly, the internal time of the moving particle considered in (2) and (3) would not coincide with the $t_{\text {local }}$ defined at a given $\xi_{0}$ when the particle propagates beyond $\xi_{0}$.

Quantum Mechanics can then be a direct consequence of such a local space-time contradiction. An extended spinorial object will be necessarily extended in time, and this property is expected to forbid continouous motion at scales close to the size of the particle. Thus, the dynamical role of the local SST can be directly at the origin of the Feynman path integral (see also subsection 4.1).

An essential question would then be that of the pre-Big Bang evolution leading to the generation of standard matter and Quantum Mechanics. If the internal vacuum dynamics is at the origin of the cosmic SST geometry, the emission of standard matter and radiation can just correspond to this expansion reflecting the fact that such a phenomenon is energetically favoured $[64,65]$.

\section{Vacuum structure and dynamics}

A nontrivial question is how to define precisely the physical vacuum. If vacuum has its own internal structure and dynamics, and if standard particles are actually vacuum excitations, how can theory and phenomenology distinguish between an object present "inside the vacuum" and an equivalent item "propagating in our Universe"? And how to define vacuum excitations?

Assuming that the physical vacuum is made of preons as already suggested in [17, 18], is it possible to unambiguously define a "matter Universe" lying on this preonic vacuum? Should one just take into account local vacuum excitations to describe this matter?

At this stage, a related question must be seriously considered: is the structure of vacuum the same in all the regions of the SST Universe corresponding to the hypersphere $|\xi|=t$ at a given $t$ ? In particular, can some parts of the global SST Universe be populated by free preons able to reach our matter Universe and to propagate in it? And how does the vacuum structure vary between the different cosmic regions of the global SST?

In a solid, photons are present through the electromagnetic interaction. But external free photons can also reach a transparent solid and propagate in it. What is the situation for "free" preons traveling in the part of the Universe associated to conventional matter and to our physical vacuum? Just as matter is not homogeneous around us and several kinds of matter exist where light can propagate, there 
is no reason to exclude the possibility that vacuum be inhomogeneous at the scale of the cosmic SST and that some preons can travel as free particles through different regions of the global SST Universe. The cosmic SST geometry seems to indicate the existence of a fundamental dynamics of the physical vacuum common to all the regions of the global Universe. But simultaneously, some differences can also exist and play a significant role, including possible local fluctuations of the expansion rate.

The study by all possible ways of the structure, dynamical properties and cosmic influence of the vacuum must be the highest priority in Particle Physics and Cosmology.

In the present situation, a practical choice can be to define the vacuum as the set of preons, including virtual ones, trapped in its internal structure. "Real" preons able to travel over long distances will then be dealt with as free particles.

The question of the local vacuum rest frame (VRF) must also be seriously considered $[17,70]$. The VRF would be basically the local rest frame of the internal vacuum structure. A natural choice seems to be suggested by cosmological observations (isotropy of the cosmic microwave background...) [56, $71]$ and can in any case be a useful starting point.

The possibility that the internal dynamics of the physical vacuum is actually leading the expansion of the Universe and that standard matter just corresponds to the energy emitted in this energetically favoured process has also been considered in previous papers, including [64, 65]. Such a scenario would be radically different from standard cosmology where conventional matter (including the dark one) is assumed to lead the Universe evolution.

\subsection{Superbradyons}

Such as introduced in $[17,18]$ and further dealt with in subsequent papers including $[14,15]$, superbradyons are the preonic constituents of the physical vacuum and the bradyons (positive mass and energy, real momentum particles) of a super-relativity (a Lorentz-like symmetry with a critical speed $c_{s} \gg c$ ). They are not tachyons, but new particles that do not obey the standard Lorentz symmetry.

What can be the properties of "free" superbradyons in our matter Universe?

In $[17,18]$ and it subsequent work, it has been systematically assumed that at a speed larger than $c$ (up to some possible small corrections), "free" superbradyons are not really stable and release "Cherenkov" radiation in the form of ordinary particles as long as such decays are kinematically allowed. A "free" superbradyon would then interact with standard matter and, in particular, undergo "Cherenkov" decays until it reaches a speed equal or close to $c$.

This is the strongest constraint (no specific refractive index, no associated speed) that can be introduced on superbradyon propagation in our Universe, even if it is simultaneously conjectured that superbradyons couple weakly to standard matter. It is also tacitly assumed that superbradyons have a specific identity as physical objects and cannot just disappear by decaying into ordinary matter.

Except for their coupling to standard matter, the "free" superbradyons just considered can in principle propagate in vacuum just like photons in a transparent crystal. They would correspond to a specific family of superbradyons, but not necessarily to all the kinds of superbadyons present in the vacuum structure, just as electrons are different from photons.

It must also be noticed that "free" superbradyons can equally increase their energy in some interactions with standard matter. Globally, their interaction with matter in our Universe will depend in particular on the matter density and distribution as well as on the nature of this matter, including dark matter of which superbradyons themselves can be a significant component [24, 63].

The question can also be raised of the possible emission of superbradyons by the physical vacuum, if the vacuum is leading the expansion of the Universe and such an expansion is energetically favoured. Cosmological equations should leave room for this kind of new physics. 
If the physical vacuum has indeed a nontrivial, preonic, internal structure, the study if its physical and cosmological implications is the most fundamental research field in the relevant domains. In this context, the search and study of "free" superbradyons is a very fundamental subject.

Clearly, the physics of superbradyons is full of unknowns and open questions. A crucial one is: how to produce, detect and identify such objects? In this respect, the possibility that CERN beams locally excite the vacuum structure leading to superbradyon emission deserves serious consideration.

Similarly, the possible implications of a superbradyonic vacuum for electronics, informatics and associated fields must be carefully studied including the role of superluminal signals.

\section{Standard QM and relativity as low-energy approximations}

What can be the validity domain of the standard principles of Physics? What is really known with the required precision? It is a fact that 13 orders of magnitude separate the highest LHC proton energies from the Planck scale. Deviations from conventional relativity and Quantum Mechanics can appear above the LHC energies and become stronger as the energy scale increases [14]. Relativity and QM can then be low-energy approximations to a deeper description of matter [17, 72].

In practice, the degree of validity of QM and relativity for ultra-high energy (UHE) particles including the observed ultra-high energy cosmic rays (UHECR) remains by now unknown $[56,73,74]$ and requires further experimental, phenomenological and theoretical work.

Present data and analyses do not allow yet to elucidate [75] if the observed fall of the UHECR spectrum $[59,60]$ is really a signature of the Greisen-Zatsepin-Kuzmin (GZK) cutoff [76, 77] or corresponds, for instance, to the maximum energies of particles emitted by astrophysical sources. New physics can also generate unconventional mechanisms able to fake the GZK cutoff [27, 73]. It therefore seems difficult from a theoretical point of view, in the present situation, to reliably interpret existing data for high-energy cosmic rays like those presented in [78, 79], [80, 81] and [82, 83]. In all cases, new generations of UHECR experiments are required.

Another serious unknown is the degree of validity and precision of models and algorithms used to describe the interactions involving UHECR [84], especially if new physics can be present and modify QFT. This situation naturally generates significant experimental uncertainties.

The properties of UHECR should be carefully studied as far as possible, including satellite experiments. A systematic search for all kinds of possible signatures of new physics is especially required, together with a study of possible connections with the basic physics involved in the early Universe dynamics. Basic ingredients of these searches must be systematic tests of Lorentz symmetry at UHE, as already suggested in 1996-97 [18, 24], together with UHE tests of all the fundamental principles of standard physics including QM [16, 27] in all possible ways.

Interactions and mixing with superbradyons can also be at the origin of deformations of relativity and QM. As already emphasized, even at low energy QM and relativity are expected to be incomplete descriptions of reality in the presence of a preonic vacuum structure [14, 15].

\subsection{Possible deformations of relativity and QM}

The subject of possible deformations of particle kinematics was already dealt with in $[18,72]$ and in subsequent work, in order to test Lorentz symmetry at very high energy.

Lorentz symmetry violation (LSV) at high energy was in particular described, assuming the existence of a local VRF, by the high-energy equation:

$$
E \simeq p c+m^{2} c^{3}(2 p)^{-1}-p c \alpha\left(p c E_{a}^{-1}\right)^{2} / 2
$$


$p$ is the momentum modulus assumed to be $\ll E_{a} c^{-1}, m$ the mass of the particle, $\alpha$ a positive constant describing the strength of the deformation and $E_{a}$ an effective fundamental energy scale.

In such a scenario, a possible negative deformation term accounting for LSV:

$$
\Delta E \simeq-p c \alpha\left(p c E_{a}^{-1}\right)^{2} / 2
$$

would become larger than the mass term $m^{2} c^{3}(2 p)^{-1}$ above the transition energy $E_{\text {trans }}$ :

$$
E_{\text {trans }} \simeq \alpha^{-1 / 4}\left(E_{a} m\right)^{1 / 2} c
$$

In its simplest form, such a mechanism can possibly suppress the GZK cutoff as suggested in [18, 72]. But more sophisticated formulations can also be considered [27, 73], leading to various possible scenarios at ultra-high energy.

In all cases, the existence of a local VRF is an essential ingredient of the pattern considered. Otherwise, if there is no privileged reference frame, suitable symmetry transformations will remove the effects of apparent deformations. In a similar way to special relativity, Quantum Mechanics, energy-momentum conservation and other particle properties can also be deformed at high energy and become an incomplete low-energy limit of a more sophisticated dynamics.

Deformed Heisenberg algebras and scenarios involving a non-commutative space-time have been considered in $[16,85]$, in $[86,87]$ and in several other approaches including strings [88, 89]. In particular, q-deformations of the quantum algebra were studied in [85].

In $[16,56]$, we considered new commutation relations between momentum variables where the commutators between components associated to different directions would vanish in the zeromomentum limit and become significant at UHE. An explicit example of the deformations of commutation relations involving momentum operators was:

$$
\begin{aligned}
\Delta p_{x} \Delta p_{y} & \gtrsim \Phi\left(p^{2}\right) \\
\Delta p_{y} \Delta p_{z} & \gtrsim \Phi\left(p^{2}\right) \\
\Delta p_{z} \Delta p_{x} & \gtrsim \Phi\left(p^{2}\right)
\end{aligned}
$$

where $x, y$ and $z$ design three orthogonal space directions, and $\Phi(0)=0$. Again, a local privileged reference frame is tacitly involved in such a formulation of Quantum Mechanics that can be associated to the explicit emergence of fundamentally new physics at energies far below Planck scale.

\section{Possible signatures of a preonic vacuum}

The direct detection of "free" superbradyons is not the only possible way to search for potential signatures of a preonic vacuum. In particular, black holes, gravitational waves and CERN experiments can provide examples of such a quest for new physics of preonic origin $[64,65]$.

The interaction with a preonic vacuum structure can modify the internal black hole dynamics. Not only the equations of standard physics would cease to hold below some critical distance scale and black hole dynamics would be modified, but a nontrivial vacuum structure may interact with the matter trapped in the black hole. Preons and other "exotic" objects released by vacuum inside the black hole or produced through interactions would potentially be able to escape the black hole region if, for instance (as in the case of superbradyons) : i) they can travel at a speed much larger than that of light ; ii) their coupling to gravitation is much smaller than that of standard matter.

Several kinds of unconventional mechanisms can be at work in such a scenario. An example is the possibility that the preonic vacuum structure absorbs matter trapped in the black hole and releases 
it outside the black hole. In this and other ways, a superbradyonic vacuum can potentially generate new forms of black hole radiation mechanisms involving higher energies than the thermal radiation considered by Stephen Hawking [90].

The recent observations $([91,92]$ and $[93,94]$ reported by the collaborations LIGO [95] and VIRGO $[96,97]$ indicate the detection of gravitational waves generated by two binary black hole mergers (GW150914 and GW151226). A careful look to the published data analyses based on standard techniques allows to better explicit the potential role of the preonic vacuum and the modifications one can expect in such an unconventional scenario.

In [97], the LIGO and VIRGO collaborations present an updated analysis of GW150914 data. The abstract of the article refers to two black holes of approximately 35 solar masses each that orbited each other as close as $\sim 350 \mathrm{Km}$ before merging to a single black hole. An attempt is presented to estimate the distance of such an event to earth and the energy radiated in the form of gravitational waves. Obviously, a preonic vacuum may have influenced the dynamics of the two black holes, as well as the merging process and the associated emission of waves.

In the introduction, the authors of [97] define the black hole as "a region of space-time where the gravitational fiel is so intense that neither matter nor radiation can escape". As an initial approximation, they refer to the standard Schwarzschild radius $r_{S c h w a r z}$ through the expression:

$$
r_{\text {Schwarz }}=2 G m c^{-2}=2.95 m M_{\odot}^{-1} \mathrm{Km}
$$

where $M_{\odot}$ is the solar mass, $m$ the mass of the black hole and $G$ the usual Newton's gravitational constant. Clearly, such an expression cannot be applied to preonic vacuum constituents present inside the black hole. Futhermore, the presence of nonstandard objects is expected to modify black hole dynamics including formulae like (11) and its more precise versions that incorporate general-relativistic effects and are also considered in [97].

In Section 1 of [97], the authors write: "During the period when gravitational wave frequency and amplitude are increasing orbital motion of two bodies is the only possible explanation". Again, alternatives to such an interpretation can be naturally generated by the interaction of the preonic vacuum with the melting black holes. The same objection can be raised when later, in Section 4 , the frequencies reached by gravitational waves are used to reject families of black hole models.

Preonic waves must also be considered as a possible source of high-frequency gravitational waves, potentially modifying their internal structure and properties in a significant way.

Simultaneously, ANTARES [98] and IceCube [99] have found no high energy neutrino event that could be in coincidence with the GW150914 signal such as reported by LIGO and VIRGO [100]. Again, preonic constituents of vacuum may have replaced the emission of standard particles in the merging black hole dynamics. In this case, they can also modify the standard timing for gravitational wave emission and propagation.

Not only a preonic vacuum would modify the black hole dynamics described in $[101,102]$ or in $[103,104]$, but it can play a strong role in primordial black holes and, more globally, in a related pre-Big Bang scenario where standard physics would be progressively generated.

\section{Gödel-Cohen incompleteness}

An introduction to Gödel's incompleteness theorems can be found in [105]. Applying it to the situation described in the present lecture is a far from trivial task.

In [106, 107], the authors explicitly claim that "for any consistent, recursive axiomatisation of mathematics, there exist specific Hamiltonians for which the presence or absence of a spectral gap is independent of the axioms". The same philosophy is developed in [108, 109]. 
But what can be the actual situation in our world if the version of Quantum Mechanics used for quantum calculations is not a consistent system of axioms but a set of approximations to the physical laws, and if the laws of Physics result from the preonic vacuum dynamics where standard particles are vacuum excitations and Quantum Mechanics is generated from such a deeper dynamics?

If Quantum Mechanics is in practice the expression of more complete laws resulting from the dynamics of a superbradyonic vacuum, is it possible to obtain a deformed, approximate version of the quantum theory for standard particles such that all relevant calculations of Quantum Physics escape the usual consequences of Gödel incompleteness?

In the presence of a preonic vacuum, no real law of Physics is expected to refer only to conventional particles. If this is the case in practical formulations, intrinsic error bars should actually be made explicit. These error bars must refer not only to standard matter itself but also to preonic objects unknown in terms of this matter. They should be present in the values of all the conventional physical quantities, including the most fundamental ones.

A new approach to the formulation of the laws of Physics is clearly required, searching in particular for a dynamical pattern where incompleteness would apply to the preonic vacuum dynamics but the approximate quantum calculations for standard matter would escape its implications using a well-suited version of Quantum Mechanics.

\section{Further dynamical and cosmological considerations}

As already discussed in previous papers [23, 26], "free" massive superbradyons with a speed close to $c$ can form a new kind of cosmic dark matter in our Universe [27, 61]. An important open question is in such case how would two "free" superbradyons interact, and what can emerge from such a process.

It also remains unclear how well "free" superbradyons would obey the approximate quantummechanical laws that describe vacuum excitations. In all cases, a "free" massive superbradyon with a speed equal to $c$ would have a rest energy $m c_{s}^{2}$ ( $m=$ inertial mass) much larger than its kinetic energy equal to $m c^{2} / 2$. Even coupling very weakly to gravitation, such an extremely large rest energy can be expected to produce significant gravitational effects. In particular, "free" massive superbradyons would in principle be trapped by strong enough gravitational fields.

The possibility that signatures of the preonic vacuum are produced at LHC or spontaneously emitted by vacuum inside this accelerator in the presence of strong particle beams should also be considered. A possible signal can be an important Cherenkov-like emission of standard particles by an object with low momentum. In a recent experimental search, the CMS collaboration has displayed [110] some atypical multi-jet events that could potentially be associated to the production of black holes. The possibility that similar events actually involve a superbradyonic component should also be studied, together with the search for possible black hole candidates.

Issues related to Quantum Gravity (QG) and to the role of strings have also been discussed in previous contributions to this Conference (see, for instance, $[19,57]$ ). Preonic and pre-Big Bang approaches, together with new space-time geometries like the SST, can radically change the situation with respect to standard theories. In practice, the notion of QG as it is usually formulated, and the Planck scale itself, would no longer make sense [26].

As strings are naturally composite objects $[16,57]$, new Regge-like and string-like structures are expected $[14,57]$ to be generated from a preonic vacuum with a spinoral space-time geometry, including descriptions of standard particles as excitations. The SST geometry can naturally lead to Regge trajectories with a spin spacing of $1 / 2$ instead of 1.

Some of the topics discussed in this lecture are dealt with in more detail in two contributions to this Conference [62]. 


\section{Conclusion and comments}

The origin of Quantum Mechanics is a crucial issue, not only for fundamental Physics but also for several fields of high-technology applications (electronics, computers [113, 114], telecommunications $[115,116] \ldots)$. If conventional particles are actually excitations of a preonic vacuum, standard laws like Quantum Mechanics and relativity are expected to be low-energy approximations to a more fundamental (preonic) dynamics. Then, Gödel-Cohen incompleteness will not apply in its usual form.

Research on Particle Physics and Cosmology is actually just beginning, and will require a very long period including new generations of experiments and observations.

If the expansion of our Universe has always been driven by the dynamics of a preonic vacuum, why did such an expansion have a previous phase different from the present one?

\section{References}

[1] See, for instance, H. Takesue et al., Quantum teleportation over $100 \mathrm{~km}$ of fiber using highly efficient superconducting nanowire single-photon detectors, Optica 2, 832 (2015), and references therein.

[2] B. Korzh et al., Provably secure and practical quantum key distribution over $307 \mathrm{~km}$ of optical fibre, Nature Photonics 9, 163 (2015), arXiv:1407.7427

[3] H.-L. Yin et al., Measurement device independent quantum key distribution over $404 \mathrm{~km}$ optical fibre, arXiv:1606.06821

[4] A. Kellerer, Quantum Telescopes, Astronomy and Geophysics, 55 (3): 3.28 (2014), arXiv:1403.6681

[5] A.R. Kurek et al., Quantum Telescopes: feasibility and constraints, Optics Letters 41 (6), 1094 (2016), arXiv: 1508.04275

[6] See, for instance, on the site of the Austrian Academy of Sciences, Fist Quantum Satellite Successfully Launched, (press release).

[7] Xinhua Insight: China launches first-ever quantum communication satellite, http://news.xinhuanet.com/english/2016-08/16/c_135601064_2.htm

[8] P. Shadbolt, J.C.F. Mathews, A. Laingand J.L. O'Brien, Testing foundations of quantum mechanics with photons, Nature Physics 10, 278 (2014) and arXiv:1501.03713

[9] B. Hensen et al., Loophole-free Bell inequality violation using electron spins separated by 1.3 kilometres, Nature 526, 682 (2015), arXiv:1508.05949

[10] B. Hensen et al., Loophole-free Bell test using electron spins in diamond: second experiment and additional analysis, arXiv:1603.05705

[11] See, for instance, M. Van Raamsdonk, Lectures on Gravity and Entanglement, arXiv:1609.00026 , and references therein.

[12] J. M. Maldacena, The large $n$ limit of superconformal field theories and Supergravity, hepth/9711200

[13] See, for instance, L. Gonzalez-Mestres, Big Bang, inflation, standard Physics... and the potentialities of new Physics and alternative cosmologies, talk given at the 4th International Conference on New Frontiers in Physics, Kolymbari, Crete, Greece, 23-30 August 2015, EPJ Web of Conferences 126, 0212 (2016), and references therein.

[14] See, for instance, L. Gonzalez-Mestres, Tests and prospects of new physics at very high energy, contribution the 3rd International Conference on New Frontiers in Physics (ICNFP 2014), Kolymbari, Crete, Greece, 28 July - 6 August 2014, EPJ Web of Conferences 95, 05007 (2015), and references therein. 
[15] See, for instance, L. Gonzalez-Mestres, Spinorial space-time and the origin of Quantum Mechanics, contribution to the 4th International Conference on New Frontiers in Physics, Kolymbari, Crete, Greece, 23-30 August 2015, EPJ Web of Conferences 126, 05006 (2016), and references therein.

[16] L. Gonzalez-Mestres, Preon models, relativity, quantum mechanics and cosmology (I), arXiv:0908.4070, and references therein.

[17] L. Gonzalez-Mestres, Properties of a possible class of particles able to travel faster than light, Proceedings of the January 1995 Moriond Workshop, Ed. Frontières, arXiv:astro-ph/9505117

[18] L. Gonzalez-Mestres, Vacuum Structure, Lorentz Symmetry and Superluminal Particles, arXiv:physics/9704017

[19] L. Gonzalez-Mestres, Pre-Big Bang, space-time structure, asymptotic Universe, 2nd International Conference on New Frontiers in Physics, Kolymbari, Crete, Greece, August 28 - September 5, 2013, EPJ Web of Conferences 71, 00063 (2014), references therein and Post Scriptum to the preprint hal-00983005.

[20] See, for instance, the 1979 Nobel lecture by Abdus Salam, and references therein.

[21] L. Gonzalez-Mestres, Cosmological Implications of a Possible Class of Particles Able to Travel Faster than Light, Proceedings of the TAUP 1995 Conference, Nucl. Phys. Proc. Suppl. 48 (1996), 131, arXiv:astro-ph/9601090

[22] L. Gonzalez-Mestres, Pre-Big Bang, vacuum and noncyclic cosmologies, 2011 Europhysics Conference on High Energy Physics, Grenoble, July 2011, PoS EPS-HEP2011 479, and references therein.

[23] L. Gonzalez-Mestres, BICEP2, Planck, spinorial space-time, pre-Big Bang, contribution the 3rd International Conference on New Frontiers in Physics, Kolymbari, Crete, Greece, August 23 - 30 , 2014, EPJ Web of Conferences 95, 03014 (2015), and references therein.

[24] L. Gonzalez-Mestres, Physical and Cosmological Implications of a Possible Class of Particles Able to Travel Faster than Light, contribution to the 28th International Conference on High Energy Physics, Warsaw 1996, arXiv:hep-ph/9610474, and references therein.

[25] L. Gonzalez-Mestres, Space, Time and Superluminal Particles, arXiv:physics/9702026

[26] L. Gonzalez-Mestres Pre-Big Bang, fundamental Physics and noncyclic cosmologies, presented at the International Conference on New Frontiers in Physics, ICFP 2012, Kolymbari, Crete, June 10-16 2012, EPJ Web of Conferences 70, 00035 (2014), and references therein. Preprint version at mp_arc 13-18.

[27] L. Gonzalez-Mestres, Cosmic rays and tests of fundamental principles, CRIS 2010 Proceedings, Nucl. Phys. B, Proc. Suppl. 212-213 (2011), 26, and references therein. The arXiv.org version arXiv:1011.4889 includes a relevant Post Scriptum.

[28] Planck Collaboration, ESA site: http://www.cosmos.esa.int/web/planck

[29] Planck Collaboration, Planck 2013 results. XXIII. Isotropy and statistics of the CMB, Astronomy and Astrophysics 571, A23 (2014), also available at arXiv:1303.5083

[30] D. Saadeh et al., How isotropic is the Universe?, Phys. Rev. Lett. 117, 131302 (2016), arXiv:1605.07178, and references therein.

[31] For an introduction to Gödel-Cohen incompleteness, see for instance J. Steinmetz, An Intuitively Complete Analysis of Godel's Incompleteness, arXiv:1512.03667

[32] J.S. Bell, On The Einstein Podolsky Rosen Paradox, Physics 1, 195 (1964). Available at the address http://cds.cern.ch/record/111654/files/vol1p195-200_001.pdf

[33] J.S. Bell, The Theory of Local Beables, in Speakable and Unspeakable in Quantum Mechanics (Cambridge Univ. Press, Cambridge, 2004), 52. 1975 CERN preprint CERN preprint TH.2053 
available at the address http://cds.cern.ch/record/980036/files/197508125.pdf

[34] See, for instance, H.M. Wiseman, The Two Bell's Theorems of John Bell, J. Phys. A: Math. Theor. 47424001 (2014), arXiv:1402.0351

[35] See, for instance, S. Boughn, A Modest View of Bell's Theorem, arXiv:1604.08529

[36] R.A. Bertlmann, John Bell and the Nature of the Quantum World, J. Phys. A: Math. Theor. 47, 424007 (2014), arxiv:1411.5322

[37] R.A. Bertlmann, Bell's Universe: A Personal Recollection, in Quantum [Un]Speakables II: Half a Century of Bell's Theorem, eds. R. A. Bertlmann and A. Zeilinger, Springer 2016, arxiv:1605.08081

[38] A. Einstein, B. Podolsky and N. Rosen, Phys. Rev. 47, 777 (1935).

[39] In his 1964 article, John Bell cites: Albert Einstein, Philosopher Scientist (Edited by P.A. Schilp), Library of Living Philosophers, Evanston, Illinois (1949), p.85.

[40] J.S. Bell, Speakable and Unspeakable in Quantum Mechanics, Cambridge University Press, Cambridge, 2004.

[41] G. Lochak, Has Bell's inequality a general meaning for hidden variable theories?, Foundations of Physics 6, 173 (1976).

[42] J.F. Clauser, M.A. Horne, A. Shimony, A. and R.A. Holt, Proposed Experiment to Test Local Hidden-Variable Theories, Phys. Rev. Lett. 23, 880 (1969). Available at the address https://www.researchgate.net/publication/228109500

[43] See also A. Asin and L. Masanes, Certified randomness in quantum physics, Nature 540, 213 (2016), and references therein.

[44] M. Giustina et al., A significant-loophole-free test of Bell's theorem with entangled photons, Phys. Rev. Lett. 115, 250401 (2015), arXiv:1511.03190

[45] L.K. Shalm et al., A strong loophole-free test of local realism, Phys. Rev. Lett. 115, 250402 (2015), arXiv:1511.03189

[46] See, for instance, A. Aspect, Bell's Theorem : The Naive View of an Experimentalist, in Quantum [Un]speakables - From Bell to Quantum information, edited by R. A. Bertlmann and A. Zeilinger, Springer (2002), arxiv:quant-ph/0402001, and references therein.

[47] See, for instance, J-A. Larsson, Loopholes in Bell Inequality Tests of Local Realism,Journal of Physics A 47, 424003 (2014), arXiv: 1407.0363 , and references therein.

[48] W. Rosenfeld et al., Event-ready Bell-test using entangled atoms simultaneously closing detection and locality loopholes, arXiv:1611.04604

[49] See, for instance, L.A. Rozema et al., Violation of Heisenberg's Measurement-Disturbance Relationship by Weak Measurements, Phys. Rev. Lett. 109, 100404 (2012), arXiv:1208.0034

[50] W. Heisenberg, Über den anschaulichen Inhalt der quantentheoretischen Kinematik und Mechanik, Zeitschfrift für Physik 43, 172 (1927).

[51] English translation from W. Heisenbeg, The Physical Content of Quantum Kinematics and Mechanics, in Quantum Theory and Measurement, Edited by J. A. Wheeler and W. H. Zurek, Princeton Univ. Press, 1984.

[52] See, for instance, J. Cen and A. Fring, Complex solitons with real energies, Journal of Physics A: Math. and Theor. 49365202 (2016], arxiv:1602.05465, and references therein.

[53] L. Gonzalez-Mestres, Quantum Mechanics and the Spinorial Space-Time, mp_arc 15-86

[54] L. Gonzalez-Mestres, Quantum Mechanics, preonic vacuum and space-time contradiction, mp_arc 15-90

[55] L. Gonzalez-Mestres, Quantum Mechanics, space-time, preons and entanglement, mp_arc 15-92 
[56] L. Gonzalez-Mestres, High-energy cosmic rays and tests of basic principles of Physics, presented at the International Conference on New Frontiers in Physics, ICFP 2012, Kolymbari, Crete, June 10-16 2012, EPJ Web of Conferences 70, 00047 (2014), and references therein. Preprint version at mp_arc 13-19.

[57] See also L. Gonzalez-Mestres, Spinorial Regge trajectories and Hagedorn-like temperatures, presented at ICNFP 2015, EPJ Web of Conferences 126, 05005 (2016).

[58] Pierre Auger Observatory, https://www.auger.org/

[59] The Pierre Auger Collaboration, The Pierre Auger Observatory Upgrade - Preliminary Design Report, arxiv:1604.03637, and references therein.

[60] The Pierre Auger Collaboration, Testing Hadronic Interactions at Ultrahigh Energies with Air Showers Measured by the Pierre Auger Observatory, Phys. Rev. Lett. 117, 192001 (2016), arxiv: 1610.08509

[61] L. Gonzalez-Mestres, Lorentz symmetry violation, dark matter and dark energy, Invisible Universe International Conference, Paris June 29 - July 3, 2009. The arXiv.org version arXiv:0912.0725 includes a relevant Post Scriptum.

[62] L. Gonzalez-Mestres, The present status of Cosmology and new approaches to particles and Cosmos and Value of H, spinorial space-time and Universe's expansion, ICNFP 2016 Conference.

[63] L. Gonzalez-Mestres, Superbradyons and some possible dark matter signatures, arXiv:0905.4146

[64] L. Gonzalez-Mestres, Can matter accelerate the expansion of the Universe? (I) (April 26, 2016), Part of a contribution to the 5th International Conference on New Frontiers in Physics, Kolymbari, Crete, Greece, July 5 - 15, 2016. Available at mp_arc 16-33.

[65] L. Gonzalez-Mestres, Cosmological implications of a preonic vacuum (I) (August 2, 2016), Part of a contribution to the 5th International Conference on New Frontiers in Physics, Kolymbari, Crete, Greece, July 5 - 15, 2016. Available at mp_arc 16-62.

[66] G. Lemaître, The Beginning of the World from the Point of View of Quantum Theory, Letter to Nature, Nature 127, 706, 9 May 1931.

[67] L. Gonzalez-Mestres, Planck data, spinorial space-time and asymptotic Universe, mp_arc 1333, and references therein.

[68] L. Gonzalez-Mestres, Spinorial space-time and privileged space direction (I), mp_arc 13-75, and references therein.

[69] L. Gonzalez-Mestres, Spinorial space-time and Friedmann-like equations (I), mp_arc 13-80, and references therein.

[70] See, for instance, L. Gonzalez-Mestres, Lorentz symmetry violation at Planck scale, cosmology and superluminal particles, talk given at COSMO-97, Ambleside September 15-19 1997, Proceedings edited by L. Rozskowski, World Scientific 1998, arXiv:physics/9712056, references therein and subsequent papers.

[71] See, for instance, the Planck Collaboration, Planck 2015 results. XVI. Isotropy and statistics of the CMB, Astronomy \& Astrophysics 594, A16 (2016), arXiv:1506.07135, and references therein.

[72] L. Gonzalez-Mestres, Absence of Greisen-Zatsepin-Kuzmin Cutoff and Stability of Unstable Particles at Very High Energy, as a Consequence of Lorentz Symmetry Violation, Proceedings of the 25th International Cosmic Ray Conference, Potchefstroomse Universiteit 1997, Vol. 6, p. 113. arXiv:physics/9705031.

[73] L. Gonzalez-Mestres, Testing fundamental principles with high-energy cosmic rays, 2011 Europhysics Conference on High Energy Physics, Grenoble, July 2011, PoS EPS-HEP2011 390, and references therein. 
[74] L. Gonzalez-Mestres, Ultra-high energy physics and standard basic principles, contribution the 2nd International Conference on New Frontiers in Physics, Kolymbari, Crete, Greece, August 28 September 5, 2013, EPJ Web of Conferences 71, 00062 (2014). See also the Post Scriptum to the preprint version, mp_arc 14-31.

[75] A.Watson, High-Energy Cosmic Rays and the Greisen-Zatsepin-Kuzmin Effect, Rept.Prog.Phys. 77 (2014) 036901, arXiv:1310.0325.

[76] K. Greisen, End to the Cosmic-Ray Spectrum? Phys.Rev.Lett. 16 (1966), 748, http://physics.princeton.edu/ mcdonald/examples/EP/greisens_prl_16_748_66.pdf

[77] G.T. Zatsepin and V.A. Kuz'min, Upper Limit on the Spectrum of Cosmic Rays, JETP Letters 4, 78

[78] The Telescope Array Collaboration, Indications of Intermediate-Scale Anisotropy of Cosmic Rays with Energy Greater Than 57 EeV in the Northern Sky Measured with the Surface Detector of the Telescope Array Experiment, Astrophysical Journal790, L21 (2014), arXiv:1404.5890.

[79] The Telescope Array Collaboration, The Energy Spectrum of Cosmic Rays above $10^{17.2} \mathrm{eV}$ Measured by the Fluorescence Detectors of the Telescope Array Experiment in Seven Years, Astropart.Phys. 80, 131 (2016), arXiv:1511.07510, and references therein.

[80] The Pierre Auger Observatory, Large scale distribution of ultra high energy cosmic rays detected at the Pierre Auger Observatory with zenith angles up to $80^{\circ}$, arXiv:1411.6953, and references therein.

[81] The Pierre Auger Collaboration, Multi-resolution anisotropy studies of ultrahigh-energy cosmic rays detected at the Pierre Auger Observatory, arXiv:1611.06812, and references therein.

[82] R. Abbasi et al., Report of the Working Group on the Composition of Ultra High Energy Cosmic Rays, Proceedings of the UHECR workshop, Springdale, USA, 2014, arXiv:1503.07540, and references therein.

[83] Telescope Array and Pierre Auger Collaborations, Pierre Auger Observatory and Telescope Array: Joint Contributions to the 34th International Cosmic Ray Conference (ICRC 2015), arXiv:1511.02103, and references therein.

[84] See, for instance, The Pierre Auger Collaboration, Testing Hadronic Interactions at Ultrahigh Energies with Air Showers Measured by the Pierre Auger Observatory, Phys. Rev. Lett. 117, 192001 (2016), arXiv:1610.08509, and references therein.

[85] J. Wess, q-Deformed Heisemberg Algebras, Lectures given at the 38. Internationale Universitätswochen für Kern- und Teilchenphysik, Schladming (Austria), January 1999, arXiv:math$\mathrm{ph} / 9910013$, and references therein.

[86] S. Majid and H. Ruegg, Bicrossproduct structure of the Poincaré group and noncommutative geometry, Physics Letters B 334, 348-354 (1994), arXiv:hep-th/9405107arXiv:hep-th/9405107.

[87] A. Connes and J. Lott, Particle models and noncommutative geometry, Nucl. Phys. Proc. Suppl. B 18, 29 (1990), http://deepblue.lib.umich.edu/bitstream/handle/2027.42/29524/0000611.pdf

[88] N.E. Mavromatos and R.J. Szabo, arXiv.org, arXiv:hep-th/9811116

[89] N. Seiberg and E. Witten, String theory and noncommutative geometry, JHEP 09, 032 (1999), arXiv:hep-th/9908142.

[90] S. Hawking, Black hole explosions?, Nature 248, 30 (1974).

[91] B.P. Abbott et al. (LIGO Scientific Collaboration and Virgo Collaboration), Observation of Gravitational Waves from a Binary Black Hole Merger, Phys. Rev. Lett. 116, 061102 (2016).

[92] B.P. Abbott et al. (LIGO Scientific Collaboration and Virgo Collaboration), Astrophysical Implications of the Binary Black-Hole Merger GW150914, The Astrophysical Journal Letters 818, L22 (2016). 
[93] B.P. Abbott et al. (LIGO Scientific Collaboration and Virgo Collaboration), Observation of Gravitational Waves from a 22-Solar-Mass Binary Black Hole Coalescence, Phys. Rev. Lett. 116, 241103 (2016).

[94] B.P. Abbott et al. (LIGO Scientific Collaboration and Virgo Collaboration), Binary Black Hole Mergers in the first Advanced LIGO Observing Run, arXiv:1606.04856

[95] The LIGO Scientific Collaboration, http://www.ligo.org/

[96] VIRGO Collaboration, http://www.virgo-gw.eu/

[97] The LIGO Scientific Collaboration, the Virgo Collaboration, The basic physics of the binary black hole merger GW150914, arXiv:1608.01940

[98] ANTARES Collaboration, http://antares.in2p3.fr/

[99] IceCube South Pole Neutrino Observatory, http://icecube.wisc.edu/

[100] ANTARES Collaboration, IceCube Collaboration, LIGO Scientific Collaboration, Virgo Collaboration, High-energy Neutrino follow-up search of Gravitational Wave Event GW150914 with ANTARES and Ice Cube, arXiv:1602.05411

[101] T. Jacobson, Introductory Lectures on Black Hole Thermodynamics, 1996 Lectures given at the University of Utrecht.

[102] G. 't Hooft, Introduction to the Theory of Black Holes, 2009 Lectures presented at the Utrecht University, ITP-UU-09/11, SPIN-09/11.

[103] L. Gualtieri and V. Ferrari, Black Holes in general Relativity, Università degli studi di Roma, 2011.

[104] Harvey Reall, Lecture notes on Black Holes (2016), University of Cambridge.

[105] Stanford Encyclopedia of Philosophy, Gödel's Incompleteness Theorems, https://plato.stanford.edu/entries/goedel-incompleteness/

[106] T. Cubitt, D. Perez-Garcia and M. Wolff, Undecidability of the Spectral Gap, Nature 528, 207 (2015), arXiv: 1502.04135 .

[107] T. Cubitt, D. Perez-Garcia and M. Wolff, Undecidability of the Spectral Gap (full version), arXiv: 1502.04573 .

[108] J. Bausch et al., Size-Driven Quantum Phase Transitions, arXiv:1512.05687.

[109] G. De las Cuevas et al., Fundamental limitations in the purifications of tensor networks, Journal of Mathematical Physics 57, 071902 (2016), arXiv:1512.05709.

[110] CMS Collaboration, Search for black holes at $\sqrt{s}=13 \mathrm{TeV}$, http://cmsresults.web.cern.ch/cms-results/public-results/preliminary-results/EXO-15-007 . See also $[111,112]$

[111] CMS Collaboration, Search for resonances and quantum black holes using dijet mass spectra in proton-proton collisions at sqrt( $s)=8$ TeV, Phys. Rev. D 91, 052009 (2015), arXiv:1501.04198

[112] CMS Collaboration, Search for lepton flavour violating decays of heavy resonances and quantum black holes to an e-mu pair in proton-proton collisions at $\operatorname{sqrt}(s)=8 \mathrm{TeV}$, The European Physical Journal C 76, 317 (2016), arXiv:1604.05239

[113] S.J. Devitt, Performing Quantum Computing Experiments in the Cloud, Phys. Rev. A 94, 032329 (2016), arXiv:1605.05709

[114] S. Boixo et al., Characterizing Quantum Supremacy in Near-Term Devices, arXiv:1608.00263

[115] See also QiChao Sun et al., Quantum teleportation with independent sources over an optical fibre network, Nature Photonics 10, 671 (2016), arXiv: 1602.07081 , and references therein.

[116] R. Valivarthi et al., Quantum teleportation across a metropolitan fibre network, Nature Photonics 10, 676 (2016), arXiv:1605.08814 